\title{
Corrections
}

\section{Correction: Seredynski et al., Neuroestrogens Rapidly Regulate Sexual Motivation But Not Performance}

In the article "Neuroestrogens Rapidly Regulate Sexual Motivation But Not Performance” by Aurore L. Seredynski, Jacques Balthazart, Virginie J. Christophe, Gregory F. Ball, and Charlotte A. Cornil, which appeared on pages 164-174 of the January 2, 2013 issue, the authors regret one error in the original Figure 6 . In Figure $6 B$, the abbreviations ICI and TMX should be ATD and VOR, respectively. This error (and its correction) does not affect the interpretation of the conclusions. The correct Figure 6 and legend are provided below.

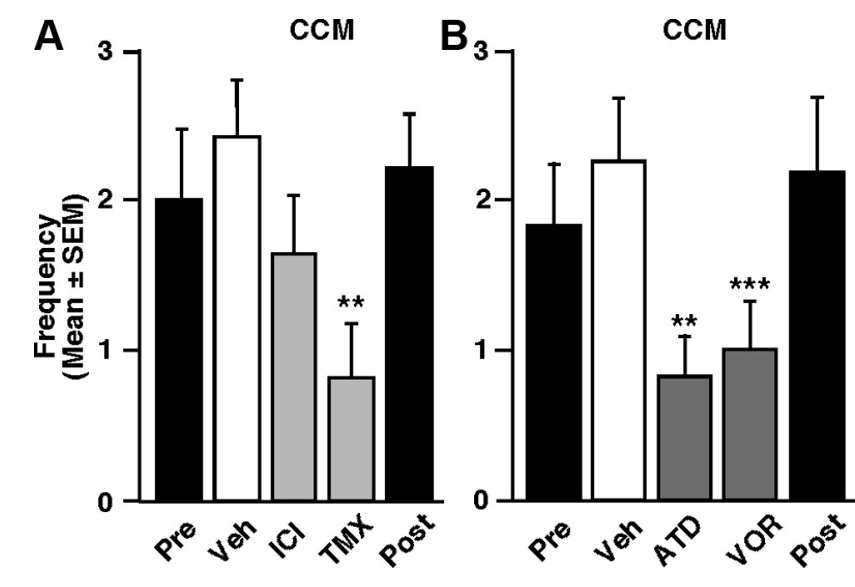

Figure 6. Effects of acute blockade of estrogen action or synthesis on copulatory behavior of behaviorally active males tested in large test chambers (group 2). The acute blockade of estrogen action by general estrogen antagonists $(\boldsymbol{A}, n=14)$ or synthesis by aromatase inhibitors ( $\boldsymbol{B}$, $n=12)$ profoundly inhibits CSB when measured in a large test arena $(90 \times 90 \times 50 \mathrm{~cm}) 30$ min after injection compared with control vehicle (Veh) injection. The "Pre" and "Post" black bars provide reference behavior frequencies after vehicle intracerebroventricular injections performed before and after the acute treatments, but these data are not included in the statistical analyses. ${ }^{* *} p<0.01$ and ${ }^{* * *} p<0.001$ versus vehicle (Vh) by Newman-Keuls post hoc tests after identification of a significant treatment effect (repeated measure) by two-way ANOVA.

DOI: $10.1523 / J N E U R O S C I .0316-13.2013$

\section{Correction: Rakhade et al., Glutamate Receptor 1 Phosphorylation at Serine 831 and 845 Modulates Seizure Susceptibility and Hippocampal Hyperexcitability after Early Life Seizures}

In the article "Glutamate Receptor 1 Phosphorylation at Serine 831 and 845 Modulates Seizure Susceptibility and Hippocampal Hyperexcitability after Early Life Seizures” by Sanjay N. Rakhade, Erin F. Fitzgerald, Peter M. Klein, Chengwen Zhou, Hongyu Sun, Richard L. Hunganir, and Frances E. Jensen, which appeared on pages 17800-17812 of the December 5, 2012 issue, the authors regret misspelling the sixth author's last name. The corrected author line is: Sanjay N. Rakhade, Erin F. Fitzgerald, Peter M. Klein, Chengwen Zhou, Hongyu Sun, Richard L. Huganir, and Frances E. Jensen, which has been corrected on the online PDF version.

DOI: 10.1523/JNEUROSCI.0559-13.2013 